\title{
Prevalência de sobrepeso e obesidade em escolares de alto nível socioeconômico em Londrina, Paraná, Brasil
}

\section{Prevalence of overweight and obesity in schoolchildren of high socioeconomic level in Londrina, Paraná, Brazil}

Enio Ricardo Vaz RONQUE ${ }^{1,2,3}$

Edilson Serpeloni CYRINO ${ }^{1}$

Valfredo Ribeiro DÓREA ${ }^{1,4}$

Helio Serassuelo JÚNIOR ${ }^{1}$

Enori Helena Gemente GALDI ${ }^{2}$

Miguel de ARRUDA²

\section{RES U M O}

\section{Objetivo}

Verificar a prevalência de sobrepeso e obesidade em escolares na faixa etária entre sete e dez anos, de ambos os sexos e de alto nível socioeconômico.

\section{Métodos}

Quinhentos e onze escolares (274 meninos e 237 meninas) foram submetidos a medidas antropométricas de massa corporal, estatura e espessuras de dobras cutâneas (tricipital e subescapular). Valores de índice de massa corporal maiores ou iguais ao percentil 85 e menores do que percentil 95 foram utilizados para a determinação de sobrepeso, ao passo que valores de índice de massa corporal maior ou igual ao percentil 95 foram adotados como indicadores de obesidade. O nível socioeconômico foi estabelecido a partir de informações produzidas por um questionário, de acordo com o grau de instrução dos pais e os bens de consumo familiar.

\section{Resultados}

A prevalência total de sobrepeso foi de 19,7\% nos meninos e 17,3\% nas meninas, sem diferenças significantes entre sexo e faixa etária $(p>0,05)$. Por outro lado, a prevalência de obesidade em meninos e meninas foi de

\footnotetext{
1 Grupo de Estudo e Pesquisa em Metabolismo, Nutrição e Exercício. Centro de Educação Física e Desportos. Universidade Estadual de Londrina. Rod. Celso Garcia Cid, km 380, Campus Universitário, 86051-990, Londrina, PR, Brasil. Correspondência para/Correspondence to: E.R.V. RONQUE. E-mail: <enioronque@sercomtel.com.br>.

2 Departamento de Ciências do Esporte, Faculdade de Educação Física, Universidade Estadual de Campinas. Campinas, SP, Brasil.

3 Grupo de Estudo e Pesquisa em Atividade Física e Exercício, Departamento de Educação Física, Universidade Estadual Paulista. Presidente Prudente, SP, Brasil.

4 Departamento de Educação, Universidade do Estado da Bahia. Teixeira de Freitas, BA, Brasil.
} 
$17,5 \%$ e $9,3 \%$, respectivamente, com diferenças significantes entre os sexos aos nove $(p<0,01)$ e dez anos $(p<0,05)$, bem como no conjunto de todas as idades $(p<0,01)$.

\section{Conclusão}

Os resultados indicam taxas de prevalência de sobrepeso ( 19\%) e de obesidade ( 14\%) bastante superiores à média da população brasileira na faixa etária entre os sete e dez anos. Portanto, diferentemente do observado em países desenvolvidos, o alto nível socioeconômico parece afetar negativamente a prevalência de sobrepeso e obesidade, aumentando os riscos para o desenvolvimento de disfunções metabólicas em idades precoces.

Termos de indexação: criança, hábitos alimentares, obesidade, prevalência.

\section{A B S T R A C T}

\section{Objective}

To verify the prevalence of overweight and obesity in 7 to 10 year old schoolchildren of both sexes and high socioeconomic level.

\section{Methods}

Five hundred and eleven schoolchildren (274 boys and 237 girls) were submitted to anthropometrical measurements of body mass, stature and subscapular and triceps skin-fold thickness. Body mass indexes $\geq 85$ th percentile and $<95$ th percentile were used to determine overweight, whereas body mass indexes $\geq 95$ th percentile were adopted as indicators of obesity. The socioeconomic level was established based on information obtained from a questionnaire, considering the degree of education of the parents and the familiar consumables.

\section{Results}

The total prevalence for overweight was $19.7 \%$ for the boys and $17.3 \%$ for the girls, with no significant differences amongst ages and sexes ( $p>0.05)$. On the other hand, the prevalence for obesity in the boys and girls was $17.5 \%$ and $9.3 \%$, respectively, with significant differences between the sexes at 9 years $(p<0.01)$ and 10 years $(p<0.05)$ of age, as well as amongst the entire group of ages $(p<0.01)$.

\section{Conclusion}

The results indicated a high prevalence ratio for overweight ( 19\%) and obesity ( 14\%), much greater than the average for the 7-10 year old Brazilian population. Therefore, different from that observed in developed countries, a high socioeconomic level seems to negatively affect the prevalence for overweight and obesity, increasing the risk of the precocious development of metabolic dysfunctions.

Indexing terms: child, food habits, obesity, prevalence.

\section{N T R O D U Ç Ã O}

O controle da obesidade tem sido, sobretudo ao longo das duas últimas décadas, um dos maiores desafios de pesquisadores e profissionais da área de saúde, uma vez que o acúmulo excessivo de gordura corporal está associado ao desenvolvimento ou agravamento de inúmeras disfunções metabólicas, tais como cardiopatias, hipertensão arterial, diabetes, hipercolesterolemia, hiperlipidemia, entre outras $^{1-4}$.

Embora muitos recursos financeiros venham sendo investidos em pesquisas e campanhas de conscientização da população para a prevenção e controle da obesidade, as perspectivas não são nada promissoras, visto que a epidemia da obesidade tem crescido assustadoramente em diferentes populações (crianças, adolescentes, adultos e idosos). Esse fenômeno tem se alastrado além dos países desenvolvidos, atingindo um contingente cada vez maior, também, dos países em desenvolvimento ${ }^{5}$, como o Brasil. Nesse sentido, de acordo com as informações apresentadas no Consenso Latino-Americano sobre Obesidade ${ }^{6}$, a prevalência de obesidade no Brasil sofreu um incremento de aproximadamente $53 \%$ entre meados da 
década de 70 e o final da década de 80 do século $X X$.

Um fato que tem preocupado os especialistas é que o desenvolvimento do sobrepeso e da obesidade tem sido constatado em idades cada vez mais precoces. Desse modo, a prevalência da obesidade na infância e na adolescência tem ocupado papel de destaque nas discussões relacionadas à saúde de jovens, motivando a realização de diversos estudos ${ }^{7-12}$, uma vez que o excesso de gordura corporal em crianças e adolescentes, assim como em populações com idades mais avançadas, pode representar um perigoso fator de risco para a saúde ${ }^{13}$.

Com relação à prevalência de obesidade infantil, o Brasil já apresentava na segunda metade do século XX (final da década de 80), índices por volta de $7 \%$ no sexo masculino e $9 \%$ no sexo feminino ${ }^{14}$. Acredita-se que atualmente essa taxa possa ter avançado perigosamente, visto o exemplo de outros países nos quais as taxas de prevalência de obesidade vêm crescendo assustadoramente nas últimas décadas ${ }^{15,16}$.

No Brasil, bem como na maioria dos países em desenvolvimento, a prevalência tanto do sobrepeso quanto da obesidade parece ser maior na população mais favorecida economicamente ${ }^{10}$, ao contrário do que ocorre nos países desenvolvidos onde a grande maioria das crianças com sobrepeso ou obesas pertence a famílias de classe socioeconômica baixa ${ }^{17}$.

Considerando que por volta de $20 \%$ da obesidade diagnosticada em indivíduos adultos parece originar-se na infância ${ }^{18}$ e que o monitoramento da quantidade de gordura corporal na população jovem pode ser um importante aliado na prevenção da saúde, o propósito do presente estudo foi verificar a prevalência de sobrepeso e obesidade em escolares na faixa etária entre sete e dez anos de idade, de ambos os sexos e de alto nível socioeconômico.

\section{MÉ T O D OS}

As informações a serem apresentadas neste estudo foram obtidas entre os meses de setembro e novembro de 2003 e fazem parte da coleta de dados inicial de um projeto de pesquisa mais amplo, de caráter longitudinal, intitulado "Análise do crescimento e da aptidão física relacionada à saúde em escolares de alto nível socioeconômico".

A população alvo deste estudo foi composta por escolares de sete a dez anos, de ambos os sexos, de alto nível socioeconômico, residentes na área urbana do município de Londrina, Paraná, Brasil. Para a seleção da amostra foi realizado, inicialmente, um levantamento para identificação do número de escolares matriculados na rede particular de ensino desse município, no ano letivo de 2002. De acordo com o setor de estatística do Núcleo Regional de Ensino da Secretaria de Educação do Estado do Paraná, estavam matriculados, somente na região urbana, 15778 estudantes, sendo 1551 no ensino pré-escolar (9,8\%); 3874 no primeiro e segundo ciclos do ensino fundamental I $(24,0 \%) ; 4134$ no terceiro e quarto ciclos do ensino fundamental II (26,2\%); 3976 no ensino médio (25,2\%); 46 no ensino normal $(0,3 \%) ; 1857$ no ensino técnico $(11,8 \%)$ e 430 no ensino supletivo $(2,7 \%)$.

Com base nessas informações, foi selecionada uma escola da região central que atendia os critérios estabelecidos para o desenvolvimento do presente estudo quanto ao nível socioeconômico, possuía um número expressivo de escolares matriculados na rede particular de ensino do município ( 15\%) e apresentava infra-estrutura adequada para a coleta de dados.

Assim, a amostra foi composta por 511 escolares (274 meninos e 237 meninas), na faixa etária dos sete aos dez anos. Portanto, considerando os dados apresentados anteriormente, foram investigados $13,2 \%$ do total de estudantes nessa faixa etária, matriculados na rede de ensino particular do município de Londrina.

Os grupos etários foram estabelecidos em idades decimais conforme os procedimentos descritos por Ross \& Marfell-Jones ${ }^{19}$. Para o agrupamento por idade, para cada sexo (Tabela 
Tabela 1. Distribuição da amostra de escolares de sete a dez anos de idade, de alto nível socioeconômico, de acordo com o sexo e a idade.

\begin{tabular}{lccc}
\hline Grupo etário & Meninos & Meninas & Total \\
\hline \pm 7 anos & 38 & 36 & 74 \\
\pm 8 anos & 86 & 61 & 147 \\
\pm 9 anos & 65 & 64 & 129 \\
\pm 10 anos & 85 & 76 & 161 \\
\hline Total & 274 & 237 & 511 \\
\hline
\end{tabular}

1), foram utilizados os intervalos de 0,50 a 0,49 convencionado com o sinal \pm , de acordo com Eveleth \& Tanner ${ }^{20}$.

Todos os responsáveis pelos escolares, após serem informados sobre o propósito dessa investigação e os procedimentos a serem adotados, assinaram um termo de consentimento livre e esclarecido. Este estudo foi desenvolvido em conformidade com as instruções contidas na Resolução 196/96 do Conselho Nacional de Saúde para estudos com seres humanos, do Ministério da Saúde, sendo aprovado pelo Comitê de Ética em Pesquisa da Universidade Estadual de Londrina.

Apesar das informações preliminares fornecidas pelo próprio estabelecimento de ensino de que a maioria dos estudantes pertencia às classes média alta e alta, para a classificação do nível socioeconômico foi aplicado um questionário desenvolvido pela Associação Brasileira de Anunciantes/Associação Brasileira dos Institutos de Pesquisa de Mercado (ABA/ABIPEME) e adaptado por Almeida \& Wickerhauser ${ }^{21}$, com escalas de classificação subdivididas em cinco categorias (A, $B, C, D, E)$, de acordo com o grau de instrução dos pais e os bens de consumo familiar. Com base nesse instrumento, todos os participantes deste estudo foram classificados como pertencentes ao nível socioeconômico elevado (categorias A e B).

A massa corporal dos sujeitos foi obtida em uma balança digital, da marca Filizolla, com precisão de $0,05 \mathrm{~kg}$, e a estatura foi determinada em um estadiômetro de madeira, com precisão de $0,1 \mathrm{~cm}$, de acordo com os procedimentos descritos por Gordon et al. ${ }^{22}$. A partir dessas medidas calculou-se o índice de massa corpóral (IMC) por meio do quociente massa corporal/ (estatura) ${ }^{2}$, sendo a massa corporal expressa em quilogramas $(\mathrm{kg})$ e a estatura em metros $(\mathrm{m})$.

Para a avaliação do comportamento da adiposidade subcutânea foram medidas as espessuras das dobras cutâneas subescapular e tricipital. Tais medidas foram realizadas por um único avaliador com um adipômetro científico da marca Lange (Cambridge Scientific Industries, Inc., Cambridge, Maryland), conforme os procedimentos descritos por Harrison et al. ${ }^{23}$. O coeficiente teste-reteste excedeu 0,95 para cada um dos pontos anatômicos com erro de medida de no máximo $\pm 1,0 \mathrm{~mm}$. Todas as medidas foram tomadas de forma rotacional e replicadas três vezes, sendo registrado o valor mediano. Com base nos valores das espessuras de dobras cutâneas, a gordura corporal relativa foi estimada por meio das equações propostas por Slaughter et al. ${ }^{24}$.

Neste estudo foram estabelecidos como pontos de corte para determinação de sobrepeso valores de IMC $\geq$ percentil 85 e < percentil 95 . Por outro lado, valores de IMC $\geq$ percentil 95 foram utilizados como indicadores de obesidade. Como padrão de referência foram adotadas as curvas de crescimento do National C'enter for Health Statistics (NCHS), revisadas recentemente (http://www.cdc.gov/growthcharts), de acordo com o sexo e a idade.

Para as comparações entre os diferentes grupos etários e de sexo, foi empregada análise de variância por dois fatores (Anova two-way). 0 teste post hoc de Scheffé, para comparações múltiplas, foi utilizado para a identificação das diferenças entre as médias. A prevalência de sobrepeso e obesidade foi determinada com base em tabelas de freqüência, sendo utilizado o teste de comparação entre proporções para a análise das diferenças de comportamento entre meninos e meninas nos diferentes grupos etários. O nível de significância estabelecido para todas as comparações foi de $p<0,05$. As informações foram tratadas no pacote computacional StatisticaTM, versão 5.1 . 


\section{RES ULTADOS}

A Tabela 2 apresenta as características antropométricas dos escolares investigados, de acordo com sexo e idade. Uma interação entre idade e sexo foi encontrada somente para a estatura $(p<0,05)$. Por outro lado, um efeito isolado tanto do sexo $(p<0,01)$ quanto da idade $(p<0,05)$ foi identificado na massa corporal e no IMC, com os meninos apresentando valores mais elevados do que as meninas. As diferenças observadas entre as crianças de sete e dez anos foram na ordem de $46,5 \%$ e $34,6 \%$, na massa corporal; $13,5 \%$ e $11,6 \%$, na estatura; e de 7,8\% e 8,4\%, no IMC; em meninos e meninas, respectivamente.
Embora nenhuma interação entre idade e sexo (Tabela 3) tenha sido identificada por Anova $(p>0,05)$, o efeito isolado da idade foi verificado nas espessuras das dobras cutâneas subescapular e tricipital, bem como nos valores da gordura corporal relativa $(p<0,01)$. As diferenças encontradas entre sete e dez anos foram na ordem de $40,8 \%$ e $32,2 \%$, na dobra subescapular; $36 \%$ e $26,9 \%$, na dobra tricipital; e de $25,8 \%$ e $22,6 \%$, na gordura corporal relativa; em meninos e meninas, respectivamente. Além disso, na dobra tricipital foi verificado, também, o efeito do sexo $(p<0,05)$, com as meninas apresentando valores superiores aos dos meninos.

Tabela 2. Características antropométricas de escolares de sete a dez anos de idade, de alto nível socioeconômico, de acordo com o sexo e a idade.

\begin{tabular}{|c|c|c|c|c|c|c|c|c|c|c|c|c|c|c|c|}
\hline \multirow{3}{*}{ Idade (anos) } & \multicolumn{6}{|c|}{ Massa corporal (kg) } & \multicolumn{3}{|c|}{ Estatura $(\mathrm{cm})$} & \multicolumn{6}{|c|}{$\mathrm{IMC}\left(\mathrm{kg} / \mathrm{m}^{2}\right)$} \\
\hline & \multicolumn{3}{|c|}{ Meninos } & \multicolumn{3}{|c|}{ Meninas } & \multicolumn{2}{|c|}{ Meninos } & Meninas & \multicolumn{3}{|c|}{ Meninos } & \multicolumn{3}{|c|}{ Meninas } \\
\hline & $M$ & \pm & $\mathrm{DP}$ & M & \pm & $\mathrm{DP}$ & M & $\pm \mathrm{DP}$ & $\pm \mathrm{DP}$ & M & \pm & $\mathrm{DP}$ & M & \pm & DP \\
\hline \pm 7 & 25,6 & \pm & 4,0 & 26,6 & \pm & 5,3 & 123,3 & $\pm 3,7$ & $125,6 \pm 4,7$ & 16,7 & \pm & 2,0 & 16,7 & \pm & 2,5 \\
\hline \pm 8 & 29,7 & \pm & 7,1 & 28,2 & \pm & 6,5 & 130,1 & $\pm 6,2$ & $127,4 \pm 5,9$ & 17,3 & \pm & 2,9 & 17,2 & \pm & 2,8 \\
\hline \pm 9 & 34,9 & \pm & 9,5 & 31,5 & \pm & 7,1 & 135,3 & $\pm 7,0$ & $133,6 \pm 6,5$ & 18,8 & \pm & 4,0 & 17,5 & \pm & 2,9 \\
\hline \pm 10 & 37,5 & \pm & 8,4 & 35,8 & \pm & 7,5 & 140,0 & $\pm 6,4$ & $140,2 \pm 7,0$ & 18,9 & \pm & 3,1 & 18,1 & \pm & 2,9 \\
\hline Fsexo & \multicolumn{6}{|c|}{$4,10 * *$} & \multicolumn{3}{|c|}{0,72} & \multicolumn{6}{|c|}{$4,28 * *$} \\
\hline Fidade & \multicolumn{6}{|c|}{$46,16^{*}$} & \multicolumn{3}{|c|}{$139,46 *$} & \multicolumn{6}{|c|}{$8,17^{*}$} \\
\hline Fsexo $\mathrm{x}$ idade & \multicolumn{6}{|c|}{1,39} & \multicolumn{3}{|c|}{3,18 ** } & \multicolumn{6}{|c|}{1,29} \\
\hline
\end{tabular}

${ }^{*} p<0,01 e^{* * 0,01<p<0,05}$.

Tabela 3. Espessura de dobras cutâneas (subescapular e tricipital) e gordura corporal relativa de escolares de sete a dez anos de idade, de alto nível socioeconômico, de acordo com o sexo e a idade.

\begin{tabular}{|c|c|c|c|c|c|c|c|c|c|c|c|c|c|c|c|c|c|c|}
\hline \multirow{3}{*}{ Idade (anos) } & \multicolumn{6}{|c|}{ Subescapular (mm) } & \multicolumn{6}{|c|}{ Tricipital (mm) } & \multicolumn{6}{|c|}{$\%$ Gordura } \\
\hline & \multicolumn{3}{|c|}{ Meninos } & \multicolumn{3}{|c|}{ Meninas } & \multicolumn{3}{|c|}{ Meninos } & \multicolumn{3}{|c|}{ Meninas } & \multicolumn{3}{|c|}{ Meninos } & \multicolumn{3}{|c|}{ Meninas } \\
\hline & $M$ & \pm & $\mathrm{DP}$ & M & \pm & $\mathrm{DP}$ & $M$ & \pm & $\overline{D P}$ & $M$ & \pm & DP & $M$ & \pm & DP & $M$ & \pm & $\mathrm{DP}$ \\
\hline \pm 7 & 7,6 & \pm & 3,4 & 8,7 & \pm & 4,2 & 11,1 & \pm & 3,3 & 13,0 & \pm & 4,3 & 17,8 & \pm & 5,2 & 19,5 & \pm & 5,8 \\
\hline \pm 8 & 8,4 & \pm & 5,0 & 9,1 & \pm & 4,7 & 12,0 & \pm & 5,1 & 13,5 & \pm & 3,9 & 18,9 & \pm & 7,5 & 20,3 & \pm & 5,7 \\
\hline \pm 9 & 11,3 & \pm & 7,6 & 10,6 & \pm & 5,9 & 15,1 & \pm & 6,8 & 14,9 & \pm & 5,3 & 23,4 & \pm & 10,7 & 22,1 & \pm & 7,1 \\
\hline \pm 10 & 10,7 & \pm & 6,5 & 11,5 & \pm & 6,3 & 15,1 & \pm & 6,0 & 16,5 & \pm & 5,2 & 22,4 & \pm & 9,4 & 23,9 & \pm & 6,9 \\
\hline Fsexo & \multicolumn{6}{|c|}{0,88} & \multicolumn{6}{|c|}{$5,25 * *$} & \multicolumn{6}{|c|}{1,42} \\
\hline Fidade & \multicolumn{6}{|c|}{$7,65^{*}$} & \multicolumn{6}{|c|}{$13,70 *$} & \multicolumn{6}{|c|}{$9,50 *$} \\
\hline Fsexo $x$ idade & \multicolumn{6}{|c|}{0,60} & \multicolumn{6}{|c|}{0,90} & \multicolumn{6}{|c|}{1,01} \\
\hline
\end{tabular}

${ }^{*} p<0,01$ e ${ }^{* *} 0,01<p<0,05$. 
Na Tabela 4 são apresentadas as taxas, absoluta e relativa, de prevalência de sobrepeso em escolares de sete a dez anos, de alto nível socioeconômico. Nenhuma diferença estatisticamente significante foi encontrada entre os sexos, na faixa etária analisada $(p>0,05)$. Embora os escores médios observados nas diferentes idades tenham sido bastante variados, verificou-se, no conjunto de todas as idades, uma elevada taxa de prevalência de sobrepeso tanto nos meninos ( 54 casos ou $19,7 \%$ ) quanto nas meninas (41 casos ou $17,3 \%)$.

Foram verificadas as taxas de prevalência de obesidade, em meninos e meninas de 7 a 10 anos (Tabela 5). A prevalência de obesidade em meninos e meninas foi de $17,5 \%$ (48 casos) e 9,3\% (22 casos), respectivamente, com diferenças significantes entre os sexos aos nove $(p<0,01)$ e 10 anos $(p<0,05)$, bem como no conjunto de todas as idades $(p<0,01)$.

Tabela 4. Prevalência (\%) de sobrepeso entre escolares de sete a dez anos de idade, de alto nível socioeconômico, de acordo com o sexo e a idade.

\begin{tabular}{lccccccc}
\hline \multirow{2}{*}{ Idade (anos) } & \multicolumn{2}{c}{ Meninos } & & \multicolumn{2}{c}{ Meninos } & \multirow{2}{*}{$p$} \\
\cline { 2 - 3 } & $\mathrm{n}$ & $\%$ & & $\mathrm{n}$ & $\%$ & \\
\hline \pm 7 & 6 & 15,8 & & 9 & 25,0 & $\mathrm{~ns}$ \\
\pm 8 & 14 & 16,3 & & 7 & 11,5 & $\mathrm{~ns}$ \\
\pm 9 & 14 & 21,5 & & 11 & 17,2 & $\mathrm{~ns}$ \\
\pm 10 & 20 & 23,5 & & 14 & 18,4 & $\mathrm{~ns}$ \\
\hline Total & 54 & 19,7 & & 41 & 17,3 & ns \\
\hline
\end{tabular}

ns= não-significante.

Tabela 5. Prevalência (\%) de obesidade entre escolares de sete a dez anos de idade, de alto nível socioeconômico, de acordo com o sexo e a idade.

\begin{tabular}{lccccccc}
\hline \multirow{2}{*}{ Idade (anos) } & \multicolumn{2}{c}{ Meninos } & & \multicolumn{2}{c}{ Meninos } & \multirow{2}{*}{$p$} \\
\cline { 2 - 3 } & $\mathrm{n}$ & $\%$ & & $\mathrm{n}$ & $\%$ & \\
\hline \pm 7 & 6 & 15,8 & & 5 & 13,9 & $\mathrm{~ns}$ \\
\pm 8 & 15 & 17,5 & & 9 & 14,8 & $\mathrm{~ns}$ \\
\pm 9 & 12 & 18,5 & & 3 & 4,7 & $<0,05$ \\
\pm 10 & 15 & 17,6 & & 5 & 6,6 & $<0,05$ \\
\hline Total & 48 & 17,5 & & 22 & 9,3 & $<0,01$ \\
\hline
\end{tabular}

ns= não-significante.

\section{I S C U S S Ã O}

Estudos de tendência secular têm indicado que atualmente crianças e adolescentes apresentam maior quantidade de gordura corporal do que seus pares de gerações passadas ${ }^{15,16,25}$. Esse fato pode estar associado a inúmeras causas, dentre as quais se destaca o aumento do balanço energético positivo, produto da redução acentuada dos níveis de atividade física diária e/ou de hábitos alimentares inadequados.

Embora muitos acreditem que os crescimentos do sobrepeso e da obesidade tenham sido causados pelo aumento do consumo de alimentos com alto valor energético e, sobretudo, ricos em lipídios e carboidratos simples, esse fator, de forma isolada, provavelmente não consiga explicar o aumento exponencial das taxas de prevalência de sobrepeso e de obesidade no mundo. A redução dos níveis de atividade física diária parece exercer, também, um papel fundamental nesse processo.

Apesar de os hábitos de vida não terem sido controlados no presente estudo, o elevado nível socioeconômico dos escolares estudados pode ter afetado negativamente as taxas de prevalência de sobrepeso (19,7\% nos meninos e $17,3 \%$ nas meninas) e obesidade $(17,5 \%$ nos meninos e 9,3\% nas meninas), uma vez que de acordo com as respostas identificadas no questionário socioeconômico aplicado, todos os escolares possuíam em suas residências mais do que um aparelho de televisão, além do que a grande maioria possuía também aparelhos de vídeo, videogames e/ou microcomputadores.

Nesse sentido, alguns estudos recentes têm indicado que muitas crianças e adolescentes têm trocado as práticas de atividades físicas de intensidade moderada ou vigorosa por atividades de baixa intensidade e, conseqüentemente, de baixo gasto energético, como assistir televisão, navegar na Internet, jogar videogame ou outros jogos eletrônicos durante as horas de lazer e tempo livre ${ }^{15,26,27}$. Desse modo, parece existir uma forte relação entre o crescimento do sobrepeso e da 
obesidade infanto-juvenil e as mudanças no estilo de vida dos jovens, visto que, via de regra, a quantidade de gordura corporal estimada é inversamente proporcional ao nível de atividade física diária realizada ${ }^{27}$.

Vale destacar que o incremento observado nos valores dos indicadores de adiposidade utilizados (espessura de dobras cutâneas), bem como nas taxas de prevalência da obesidade, com o avançar da idade, tanto em meninos quanto em meninas, sugere que esse comportamento possa ser ainda mais agravado na adolescência e na idade adulta. Esse fato fortalece a hipótese de que um adolescente obeso possa ser identificado ainda na infância, uma vez que uma criança obesa na infância pode apresentar entre 68\% e 77\% de probabilidade de permanecer obesa durante a adolescência ${ }^{28}$.

Além disso, acredita-se que a prevalência da obesidade na adolescência possa responder por cerca de $30 \%$ a $50 \%$ da obesidade na idade adulta, favorecendo o crescimento das taxas de morbidade e mortalidade nessa população ${ }^{29}$.

Por outro lado, a literatura tem indicado a presença de dimorfismo sexual, com relação aos depósitos de gordura corporal, somente a partir da puberdade, com as moças apresentando valores superiores aos dos rapazes ${ }^{17}$. Essas informações vão ao encontro dos resultados do presente estudo, que não constatou diferenças significativas na gordura corporal relativa entre os sexos, dos sete aos dez anos.

Entretanto, quando analisadas as taxas de prevalência de obesidade verificou-se a presença de dimorfismo sexual, com os rapazes apresentando escores absolutos e relativos superiores aos das moças, a partir dos nove anos e no conjunto de todas as idades. Todavia essas informações devem ser analisadas com certa cautela, uma vez que a identificação de obesidade em crianças e adolescentes por meio dos valores de IMC $\geq$ percentil 95, de acordo com as curvas de crescimento do NCHS, tem sido questionada 4,30 .

Nesse sentido, Sardinha et al. ${ }^{30}$, assumindo os pontos de corte estabelecidos por Williams et al. ${ }^{4}$ para a definição de obesidade $(\geq 25 \%$ e $\geq 30 \%$ de gordura corporal relativa para meninos e meninas, respectivamente), analisaram o potencial discriminatório do IMC, da espessura da dobra cutânea tricipital e do perímetro de braço para identificação de obesidade em crianças e adolescentes de 10 a 15 anos, de ambos os sexos. Para tanto, o método de referência utilizado foi absortometria radiológica de dupla energia (Dexa). Esse método tem sido apontado por diversos pesquisadores como um método adequado para avaliação da composição corporal em crianças e adolescentes, visto que é um modelo de três compartimentos no qual informações sobre a massa de gordura, massa isenta de osso e gordura e o conteúdo mineral ósseo podem ser acessadas com boa confiabilidade.

De acordo com os resultados relatados nesse estudo ${ }^{30}$, além do IMC apresentar um potencial discriminatório relativamente limitado para a identificação dos casos de obesidade, as informações produzidas na faixa etária dos dez aos onze anos indicaram maiores taxas de erro de predição nas meninas (30\%) do que nos meninos (18\%). Com base nessas informações, embora as idades de sete a nove anos não tenham sido investigadas, acredita-se que as diferenças nas taxas de prevalência de obesidade, na comparação entre os sexos, encontradas no presente estudo possam ser atribuídas, pelo menos em parte, às limitações do indicador utilizado (IMC).

\section{O N CLUS Ã O}

Os resultados encontrados pelo presente estudo indicaram uma taxa de prevalência de sobrepeso ( 19\%) e obesidade ( 14\%) bastante superior à média da população brasileira de sete a dez anos.

Assim, diferente do observado em países desenvolvidos, o alto nível socioeconômico parece afetar negativamente a prevalência de sobrepeso e obesidade, aumentando os riscos para o 
desenvolvimento de disfunções metabólicas em idades precoces.

Os achados deste estudo reforçam a necessidade de implementação de programas de reeducação alimentar e de prática de atividade física relacionados à promoção da saúde já nas séries iniciais do ensino fundamental. Outras investigações que incorporem informações sobre nível de atividade física habitual e hábitos alimentares em escolares de diferentes níveis socioeconômicos podem contribuir para uma análise mais criteriosa do processo de desenvolvimento do sobrepeso e obesidade em populações jovens.

\section{RE FERÊ N C I A S}

1. Ball GDC, MacCargar LJ. Childhood obesity in Canada: a review of prevalence estimates and risk factors for cardiovascular diseases and type 2 diabetes. Can J Appl Physiol. 2003; 28(1):117-40.

2. Cole TJ, Bellizzi MC, Flegal KM, Dietz WH. Establishing a standard definition for children overweight and obesity worldwide: international survey. BMJ. 2000; 302(7244):1-6.

3. Hopper CA, Gruber MB, Munoz KD, MacConnie SE, Pfingston YM, Nguyen K. Relationship of blood cholesterol to body composition, physical fitness, and dietary intake measures in third-grade children and their parents. Res Q Exerc Sport. 2001; 72(2):182-8.

4. Williams DP, Going SB, Lohman TG, Harsha DW, Srinivasan SR, Webber LS, et al. Body fatness and risk for elevated blood pressure, total cholesterol, and serum lipoprotein ratios in children and adolescents. Am J Public Health. 1992; 82(3): 358-63.

5. De Onis M, Blossner M. Prevalence and trends of overweight among preschool children in developing countries. Am J Clin Nutr. 2000; 72(4):1032-9.

6. Consenso Latino Americano sobre Obesidade [citado em 2004 mar 16]. Disponível em: http:// www.abeso.org.br/pdf/consenso.pdf

7. Abrantes MM, Lamounier JA, Colosimo EA. Prevalência de sobrepeso e obesidade em crianças e adolescentes das Regiões Sudeste e Nordeste do Brasil. J Pediatr. 2002; 78(4):335-40.

8. Barbeau P, Gutin B, Litaker M, Owens S, Riggs S, Okuyama T. Correlates of individual differences in body-composition changes resulting from physical training in obese children. Am J Clin Nutr. 1999; 69(4):705-11.

9. Chinn S, Rona RJ. Prevalence and trends in overweight and obesity in three cross-sectional studies of British children. BMJ. 2001; 322(7277):24-6.

10. Leão LSCS, Araújo LMB, Moraes LTLP, Assis AM. Prevalência de obesidade em escolares de Salvador, Bahia. Arq Bras Endocrinol Metab. 2003; 47(2):151-7.

11. Magalhães VC, Mendonça GAS. Prevalência e fatores associados a sobrepeso e obesidade em adolescentes de 15 a 19 anos das regiões Nordeste e Sudeste do Brasil, 1996 a 1997. Cad Saúde Pública. 2003; 19(Sup 1):129-39.

12. Monteiro POA, Víctora CG, Barros FC, Tomasi E. Diagnóstico de sobrepeso em adolescentes: estudo do desempenho de diferentes critérios para o índice de massa corporal. Rev Saúde Pública. 2000; 34(5):506-13.

13. Barlow SE, Dietz WH. Obesity evaluation and treatment: Expert Committee Recommendations. Pediatrics. 1998; 102(3):E29.

14. Instituto Nacional de Alimentação e Nutrição. Pesquisa Nacional sobre Saúde e Nutrição. Brasília: Ministério da Saúde do Brasil; 1989.

15. Berkey CS, Rockett HR, Field AE, Gillman MW, Frazier $\mathrm{AL}$, Camargo CA Jr, et al. Activity, dietary intake, and weight changes in a longitudinal study of preadolescent and adolescent boys and girls. Pediatrics. 2000; 105(4):E56.

16. Bundred P, Kitchiner D, Buchan I. Prevalence of overweight and obese children between 1989 and 1998: population based series of cross sectional studies. BMJ. 2001; 322(7282):1-4.

17. Malina RM, Bouchard C, Bar-Or O. Growth, maturation, and physical activity. 2nd ed. Champaign: Human Kinetics Books; 2004.

18. Fulton JE, McGuire MT, Caspersen CJ, Dietz WH. Interventions for weight loss and weight gain prevention among youth. Sports Med. 2001; 31(3):153-65.

19. Ross WD, Marfell-Jones MJ. Kinanthropometry. In: MacDougall JD, Wenger HA, Green HJ, editors. Physiological testing of the elite athlete. Ottawa: Mutual; 1982. p.75-115.

20. Eveleth PH, Tanner JM. Worldwide variation in human growth. Cambridge: Cambridge University Press; 1976.

21. Almeida PM, Wickerhauser H. O critério AbaAbipeme: em busca de uma atualização. São Paulo: Abipeme; 1991. 
22. Gordon CC, Chumlea WC, Roche AF. Stature, recumbent length, and weight. In: Lohman TG, Roche AF, Martorell R, editors. Anthropometric standardization reference manual. Champaign: Human Kinetics Books; 1988. p.3-8.

23. Harrison GG, Buskirk ER, Carter LJE, Johnston FE, Lohman TG, Pollock ML, et al. Skinfold thickness and measurement technique. In: Lohman TG, Roche AF, Martorell R, editors. Anthropometric standardization reference manual. Champaign: Human Kinetics Books; 1988. p.55-80.

24. Slaughter MH, Lohman TG, Boileau RA, Horswill CA, Stillman RJ, Van Loan MD, et al. Skinfold equations for estimation of body fatness in children and youth. Hum Biol. 1988; 60(5): 709-23.

25. Post $C L$, Víctora CG, Barros FC, Horta BL, Guimarães PRV. Desnutrição e obesidade infantis em duas coortes de base populacional no Sul do Brasil: tendências e diferenciais. Cad Saúde Pública. 1996; 12(Sup 1):49-57.

26. Andersen RE, Crespo CJ, Bartlett SJ, Cheskin LJ, Pratt M. Relationship of physical activity and television watching with body weight and level of fatness among children: results from the Third National Health and Nutrition Examination Survey. JAMA. 1998; 279(12):938-42.

27. Ball EJ, O'Connor J, Abbott R, Steinbeck KS, Davies PS, Wishart $C$, et al. Total energy expenditure, body fatness, and physical activity in children aged 6-9 y. Am J Clin Nutr. 2001; 74(4):524-8.

28. Zack PM, Harlan WR, Leaverton PE, Cornoni-Huntley J. A longitudinal study of body fatness in childhood and adolescence. Pediatrics. 1979; 95(1):126-30.

29. Dietz WH. Childhood weight affects adult morbidity and mortality. J Nutr. 1998; 128(Suppl 2):411S-4S.

30. Sardinha LB, Going SB, Teixeira PJ, Lohman TG. Receiver operating characteristic analysis of body mass index, triceps skinfold thickness, and arm girth for obesity screening in children and adolescents. Am J Clin Nutr. 1999; 70(6):1090-5.

Recebido para publicação em 29 de junho de 2004 e aceito em 30 de março de 2005. 